\title{
Human Lymphocyte Antigen B-Associated Transcript 2, 3, and 5 Polymorphisms and Haplotypes are Associated With Susceptibility of Kawasaki Disease and Coronary Artery Aneurysm
}

\author{
Yao-Yuan Hsieh, ${ }^{1,2}$ Ying-Ju Lin, ${ }^{3-5}$ Chi-Chen Chang, ${ }^{2}$ Da-Yuan Chen, ${ }^{4}$ Chin-Mu Hsu, ${ }^{4}$ \\ Ming-Min Lo, ${ }^{6}$ Kung-Hao Hsu, ${ }^{2,7}$ and Fuu-Jen Tsai, ${ }^{5,7,8 *}$ \\ ${ }^{1}$ School of Chinese Medicine, College of Chinese Medicine, China Medical University, Taichung, Taiwan \\ ${ }^{2}$ Division of Infertility Clinic, Hsieh Yao-Yuan Womens' Hospital, Taichung, Taiwan \\ ${ }^{3}$ Graduate Institute of Chinese Medical Science, China Medical University, Taichung, Taiwan \\ ${ }^{4}$ Department of Medical Research, China Medical University Hospital, Taichung, Taiwan \\ ${ }^{5}$ Department of Medical Genetics, China Medical University Hospital, Taichung, Taiwan \\ ${ }^{6}$ Enterprise Training Courses Center, Chaoyang University of Technology, Taichung, Taiwan \\ ${ }^{7}$ Department of Biotechnology and Bioinformatics, Asia University, Taichung, Taiwan \\ ${ }^{8}$ School of Post-Baccalaureate Chinese Medicine, College of Chinese Medicine, \\ China Medical University, Taichung, Taiwan
}

\begin{abstract}
Capsule: HLA-B associated transcript (BAT) 2, 3, and 5 polymorphisms and haplotypes are associated with Kawasaki disease (KD) and coronary artery aneurysm (CAA) formations. Objective: KD, an acute vasculitis with unknown etiology, involves a complex interaction of immuno-inflammatory process, cytokines activation, and genetic factors. We aimed to investigate if genetic variants of human lymphocyte antigen (HLA)-BAT2, 3 , and 5 (BAT2, 3, and 5) could be used as markers of susceptibility in KD and CAA. Methods: Individuals were divided into three groups: (1) normal controls; (2) KD with CAA; and (3) KD without CAA. Polymorphisms for BAT2 (-8671, 16483), BAT3 $(8854,2-24)$, and BAT5 (22655, 9569) were genotyped by PCR system with TaqMan allelic discrimination assay. Genotype/allelic frequencies and haplotypes (BAT2 ${ }^{-8671}$-BAT2 $2^{16483}$ BAT3 $^{8854}-$ BAT3 $^{2-24}-$ BAT5 ${ }^{22655}$-BAT5 ${ }^{9569}$ ) in
\end{abstract}

each group were compared. Results: Genotype distribution and allele frequency of BAT2 -8671, BAT3 8854, and BAT5 22655, 9569 polymorphisms in each group were significantly different. BAT2 $-8671^{\star} \mathrm{G}$, BAT3 $8854^{\star} \mathrm{C}$, BAT5 $22655^{\star} \mathrm{C}$, and $9569^{*} \mathrm{~A}$-related genotypes and alleles are correlated with the developments of $\mathrm{KD}$ and CAA. BAT haplotypes of ATTGTG and ATCATG are associated with higher susceptibilities of $\mathrm{KD}$ with CAA susceptibility. Conclusion: BAT2 -8671, BAT3 8854, and BAT5 22655, 9569 polymorphisms as well as BAT haplotypes (ATTGTG and ATCATG) might be associated with higher KD susceptibility and CAA formation. HLA-B region polymorphisms might contribute to the pathogenesis of KD and CAA. J. Clin. Lab. Anal. 24:262-268, $2010 . \quad$ (c) 2010 Wiley-Liss, Inc. disease; polymorphism

\section{INTRODUCTION}

Kawasaki disease (KD), a complex vasculitis, is associated with immunologic and genetic changes. It is characterized by persistent fever, non-purulent conjunctivitis, oropharyngeal inflammation, induration and erythema of hands and feet, rash, and cervical lymphadenopathy.
Yao-Yuan Hsieh and Ying-Ju Lin have contributed equally to this survey.

*Correspondence to: Fuu-Jen Tsai, Department of Medical Genetics, China Medical University Hospital, No.2 Yuh-Der Road, Taichung, Taiwan. E-mail: d0704@mail.cmuh.org.tw

Received 2 August 2009; Accepted 24 May 2010

DOI $10.1002 /$ jcla.20409

Published online in Wiley InterScience (www.interscience.wiley.com). 
Despite its intensive researches, the etiology of KD remains unclear. Current theories suggest that KD is an immunologically mediated vasculitis (1). The features of $\mathrm{KD}$ are immune activation and cytokine-mediated generalized vasculitis. The injured vascular tissues show subendothelial edema, vascular damage, gap formation, and fenestration of endothelial cells, which contribute to the pathogenesis of this disorder (2).

Vascular endothelial cells express major histocompatibility complex (MHC) molecules on their surface for presenting antigenic peptides to $\mathrm{T}$ cells, which initiate acquired immune responses (3). Human lymphocyte antigen (HLA) from the MHC has been reported to be associated with immune-mediated vascular diseases (4). Recently, with the clinical application of molecular genetic technology, some HLA polymorphisms have been identified to be associated with $\mathrm{KD}$. It is also believed that HLA has regulatory functions upon the innate and adaptive immune responses. The vascular endothelium is a functional barrier between the vessel wall and the blood stream. The endothelial cell damage or vascular injury leads to the expression and release of HLA-E molecules (5).

A large number of genes within HLA class I region have been identified. These genes include HLA-B associated transcript (BAT) genes. HLA class I genes have been demonstrated to be associated with $\mathrm{KD}$ development (6). However, few investigators demonstrated the association between HLA-BAT2, 3, and 5 (BAT2, 3, and 5) and KD susceptibility. In this study, we aimed to search for BAT genes that influence the susceptibility to KD and the occurrence of coronary artery aneurysms (CAAs) in Taiwanese children. Six genetic variations and related haplotypes within BAT region were evaluated. To the best of our knowledge, this is the first survey in the related aspect.

\section{MATERIALS AND METHODS}

Taiwanese children with and without the histories of KD were recruited. All individuals were divided into: (1) normal controls; (2) KD with CAA; and (3) KD without CAA. The children with KD were evaluated at China Medical University Hospital and met the criteria of KD. Every patient underwent regular echocardiography examinations. The CAA was identified of $3 \mathrm{~mm}$ dilated coronary artery in younger children ( $\leq 5$ years) or $4 \mathrm{~mm}$ dilation in older children ( $>5$ years) (7). The control group consisted of healthy children randomly selected from the Han Chinese Cell and Genome Bank (8). Control subjects were matched for sex and age with the study patients. The estimated prevalence of $\mathrm{KD}$ is less than 1/1,000 children; therefore, it should be assumed that there were no KD cases in the control group. This series was approved by the ethical committee and institutional review board of China Medical University Hospital. Informed consents were signed by the children or their parents who donated their blood.

All individuals accepted the peripheral blood samplings for genotype analyses. Genomic DNA was extracted from peripheral blood leukocytes according to standard protocols (Roche Genomic DNA kit, Roche Diagnostics, Indianapolis, USA). The single nucleotide polymorphisms (SNPs) discovery and genotyping for BAT2, 3, and 5 were obtained from dbSNP (http://ncbi. nih.gov/SNP/) and Applied Biosystems (http://www. appliedbiosystems.com) (Table 1, Fig. 1). The BAT2, 3, and 5 polymorphisms was detected by polymerase chain reaction (PCR) system with TaqMan allelic discrimination assay (Applied Biosystems, Foster City, CA, USA) (Table 1) (9). Briefly, the PCR was carried out in a total volume of $12.5 \mu \mathrm{l}$ using the following amplification protocol: denaturation at $95^{\circ} \mathrm{C}$ for $10 \mathrm{~min}$, followed by 40 cycles of denaturation at $95^{\circ} \mathrm{C}$ for $15 \mathrm{sec}$, annealing at $60^{\circ} \mathrm{C}$ for $15 \mathrm{sec}$, and extension at $72^{\circ} \mathrm{C}$ for $15 \mathrm{sec}$. Furthermore, genotyping of each sample was automatically attributed by measuring the allele-specific fluorescence on the ABI Prism 7700 Sequence Detection System, using the SDS 1.9.1 software for allelic discrimination (Applied Biosystems). Genotype frequencies of each SNP were determined by direct counting. The resulting spectra were processed with SpectroTyper (Sequenom, San Diego, CA, USA) software.

The genotype frequency and allelic frequency distributions of these polymorphisms in each group were estimated and analyzed. BAT haplotypes (BAT2 ${ }^{-8671}$ BAT2 ${ }^{16483}$-BAT3 ${ }^{8854}$-BAT3 $3^{2-24}$-BAT5 $5^{22655}$-BAT5 ${ }^{9569}$ ) in each group were compared. Haplotypes were estimated in compound heterozygotes using the PHASE program. The SAS system with $\chi^{2}$ test was utilized for statistical analyses. Allelic frequencies were expressed as a percentage of the total number of alleles. Odds ratios were calculated from genotype and allelic frequencies with $95 \%$ confidence interval. A $P$-value $<0.05$ was considered statistically significant.

\section{RESULTS}

Genotype distributions and allele frequencies of BAT2, 3, and 5 polymorphisms in each group were significantly different (Table 2). Genotype distribution and allele frequency of BAT2 -8671 polymorphism in each group were significantly different. Proportions of BAT2 $-8671 * \mathrm{G}$ homozygote/heterozygote/A homozygote in (1) controls; (2) KD with CAA; and (3) KD without CAA were: (1) $16.2 / 46.2 / 37.6 \%$; (2) $0 / 35.7 /$ $64.3 \%$; and (3) $1.7 / 69.5 / 28.8 \%$, respectively (Table 2). BAT2 $-8671 *$ G-related genotypes and alleles are 
Hsieh et al.

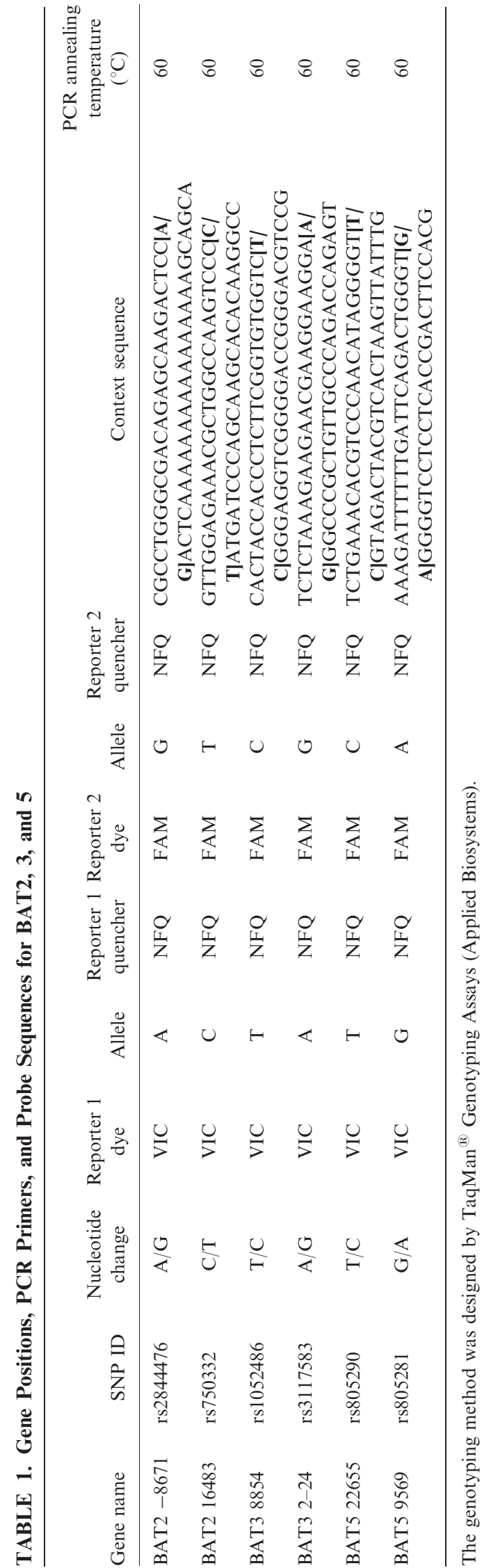

associated with susceptibility of KD and related CAA formation. In contrast, BAT2 $16483 \mathrm{~T} / \mathrm{C}$ genotypes were not associated with susceptibilities of KD and CAA.

Genotype distribution and allele frequency of BAT3 8854 polymorphism in each group were significantly different. Proportions of BAT3 $8854 *$ C homozygote/ heterozygote/T homozygote were: (1) 39.5/44.9/15.6\%; (2) $64.3 / 28.6 / 7.1 \%$; and (3) $27.9 / 60.7 / 11.5 \%$, respectively (Table 2). BAT3 $8854 *$ C-related genotypes and alleles are associated with higher susceptibility of $\mathrm{KD}$ and CAA. In contrast, BAT3 2-24 G/A genotypes were not associated with susceptibilities of KD and CAA. Genotype distribution and allele frequency of BAT5 22655 and 9569 polymorphisms in each group were significantly different. Proportions of BAT3 $22655 * \mathrm{C}$ homozygote/heterozygote/T homozygote were: (1) $42 /$ $41.7 / 16.3 \%$; (2) 60/33.3/6.7\%; and (3) $28.6 / 60.3 / 11.1 \%$, respectively (Table 2). Proportions of BAT3 9569*A homozygote/heterozygote/G homozygote were: (1) 40.8/ $43.6 / 15.6 \%$; (2) $56.7 / 36.7 / 6.7 \%$; and (3) $28.6 / 60.3 /$ $11.1 \%$, respectively.

We also observed the associations of BAT haplotypes and KD development. BAT haplotype ATTGTG and ATCATG are associated with higher susceptibilities of KD and CAA (Table 3). Other BAT haplotypes (GTTGTG, GTTATG, GTCACA, GCTATG, GCTATA, GCTACA, and ATTATG) seemed non-associated with KD or CAA susceptibilities.

\section{DISCUSSION}

$\mathrm{KD}$, an acute, self-limited, and systemic vasculitis, is one of the leading causes of acquired heart disease in children (10). Although KD is a mysterious disease of unknown etiology and pathogenesis, it is believed to be caused by infectious agents, host immune dysregulation, and genetic susceptibility (11). The development of KD involves complex interactions between immuno-inflammatory process, cytokines activation, and genetic factors. Indeed, KD is a multisystemic disorder with a possible underlying pathology of immune-mediated vasculitis (10).

Recent studies suggest a potential role of bacterial toxins in the immunopathogenesis of KD (12). During the acute stage of $\mathrm{KD}$, activation of vascular endothelial cells and increased serum levels of proinflammatory cytokines might be involved in the occurrence of inflamed and injured vessels (13). The vascular inflammation may cause the development of CAA and cardiac complications. Patients with these cardiovascular complications are at increased risk of ischemic heart disease, which may lead to myocardial infarction and sudden death (14). Although the administration of immunoglobulin significantly reduces the development of CAAs, 


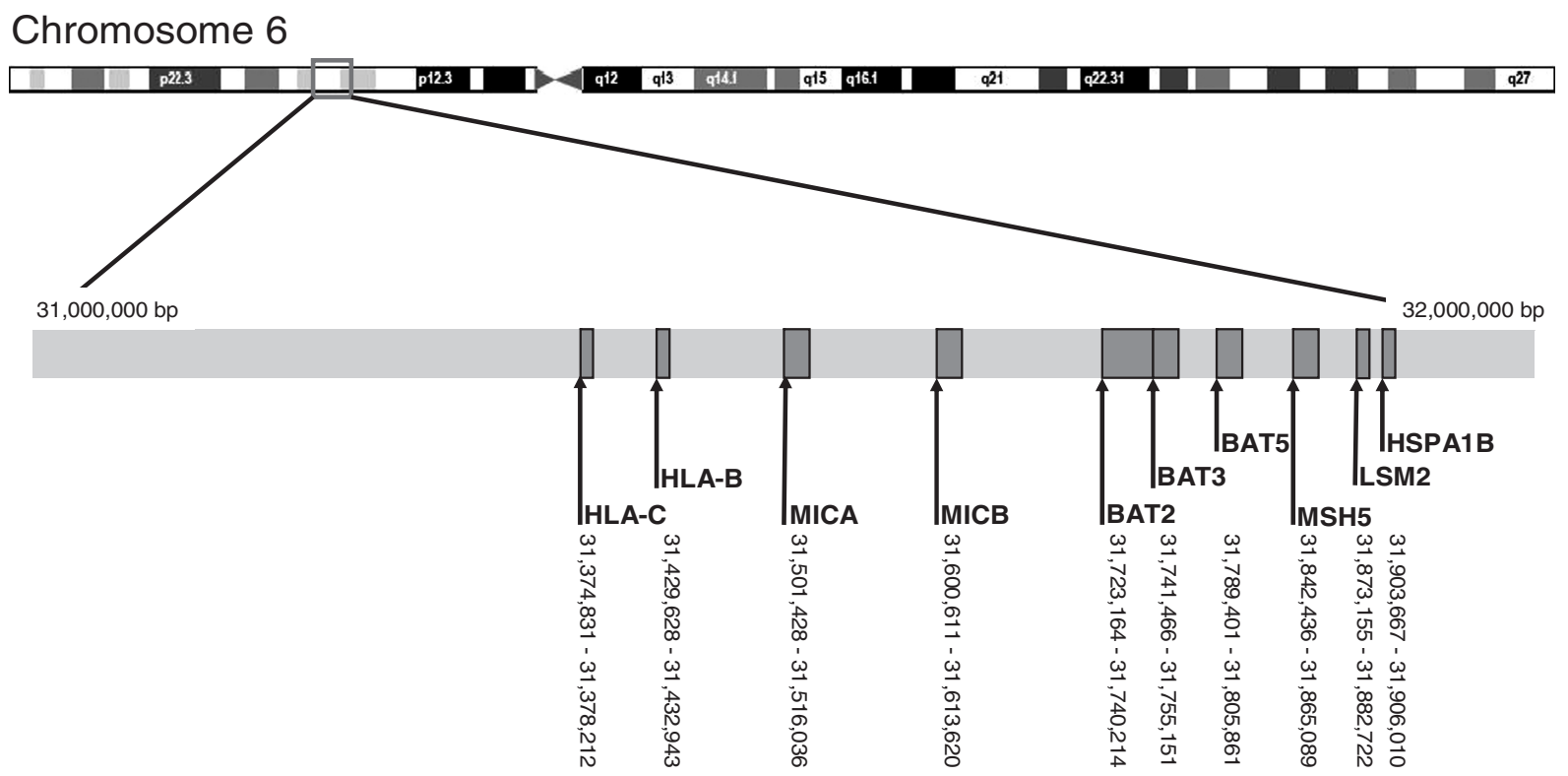

Fig. 1. Map of the BAT2, 3, and 5 located within $4 \mathrm{Mb}$ of the MHC region (from 29,900,000 to 33,900,000 bp on chromosome 6). Positions of HLA-B, C, MICA, B, BAT2, 3, and 5, MSH5, LSM2, and HSPA1B lying within this region are indicated.

around $2-15 \%$ of KD patients still suffer from this related complication of CAA (15).

Recent studies have shown that the expression of soluble HLA might have important implications upon the pathogenesis of immune-mediated vascular diseases (5). The HLA-B35, B75, and Cw09 are associated with KD susceptibilities (16). HLA-B*5801 allele might present as a genetic marker for severe cutaneous adverse reactions caused by allopurinol (8). The HLA-DR gene variations were not associated with the susceptibility of KD and CAAs in Taiwanese population (8). HLA-G plays a crucial role for the susceptibility to KD and CAA (17). MHC class II was not associated with the pathogenesis of KD (18). In our earlier survey, we also observed the association between HLA-E gene polymorphism and KD (12).

The gene coding for BAT lies adjacent to TNF gene in the central MHC region. BAT1, a member of the BAT family, could regulate the production of inflammatory cytokines and the development of neurological disorders (19). BAT1 plays a potential role for the regulation of inflammatory reactions in Alzheimer's disease pathology (19). BAT1 is also associated with chronic Chagas cardiomyopathy (20). BAT2 polymorphism has been found to be associated with severe malaria (21) and also with asthma (22), rheumatoid arthritis (23), etc.

BAT3, a nuclear protein, encodes a large proline-rich protein with unknown function. BAT3 was originally identified as one of the genes located within MHC. BAT3 is capable of modulating the transforming growth factor (TGF) signaling. BAT3 also acts as a positive regulator of TGF of collagen expression (24). The intracellular protein of BAT3 is involved in DNA damage-induced apoptosis (25). BAT3 could bind directly to NKp30 and engage NKp30 on NK cells. BAT3 also triggered NKp30-mediated cytotoxicity, which was essential for tumor rejection (26). BAT3 is an essential regulator of p53-mediated responses to genotoxic stress (27). BAT3 controls DNA damageinduced acetylation of p53 (27).

Genetic studies of the multifactorial diseases, such as $\mathrm{KD}$, are difficult to approach owing to the uncertainty of polygenic traits. The SNPs are the most abundant types of DNA sequence variation in human genome (28). The SNP markers provide a new way for the identification of complex gene-associated diseases. In this study, the genotype distributions, allelic frequencies, and haplotypes for BAT2, 3, and 5 polymorphisms in KD and non-KD patients were statistically different. BAT2 $-8671 * \mathrm{~A}, \mathrm{BAT} 38854 * \mathrm{C}$, and BAT5 $22655 * \mathrm{C}$ and $9569 *$ A related genotypes and alleles are correlated with the developments of $\mathrm{KD}$ and CAA. We also observed the BAT haplotypes, ATTGTG and ATCATG, were associated with higher susceptibilities of KD or CAA. Our findings suggested that BAT2, 3, and 5 polymorphisms are useful genetic markers in the prediction of the susceptibility of KD. It also suggests that HLA-B gene and BAT2, 3, and 5 polymorphisms might be involved in KD susceptibility and CAA development. These SNPs might influence the production of soluble HLA-B by vascular endothelial cells, which further compromise the illness progressions of KD.

In conclusion, our survey suggests that BAT2, 3, and 5 molecules might be involved in the pathogenesis of 
266 Hsieh et al.

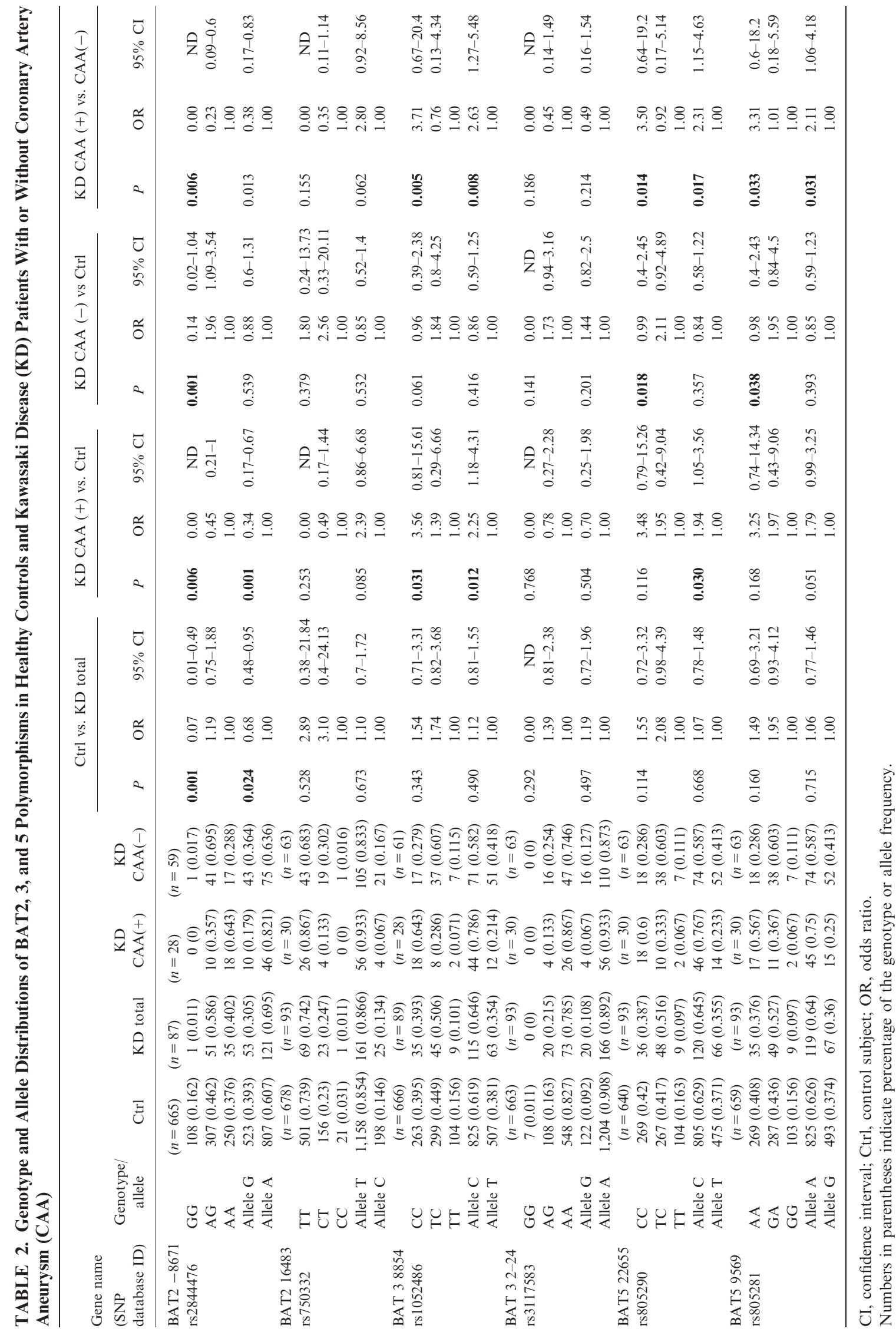




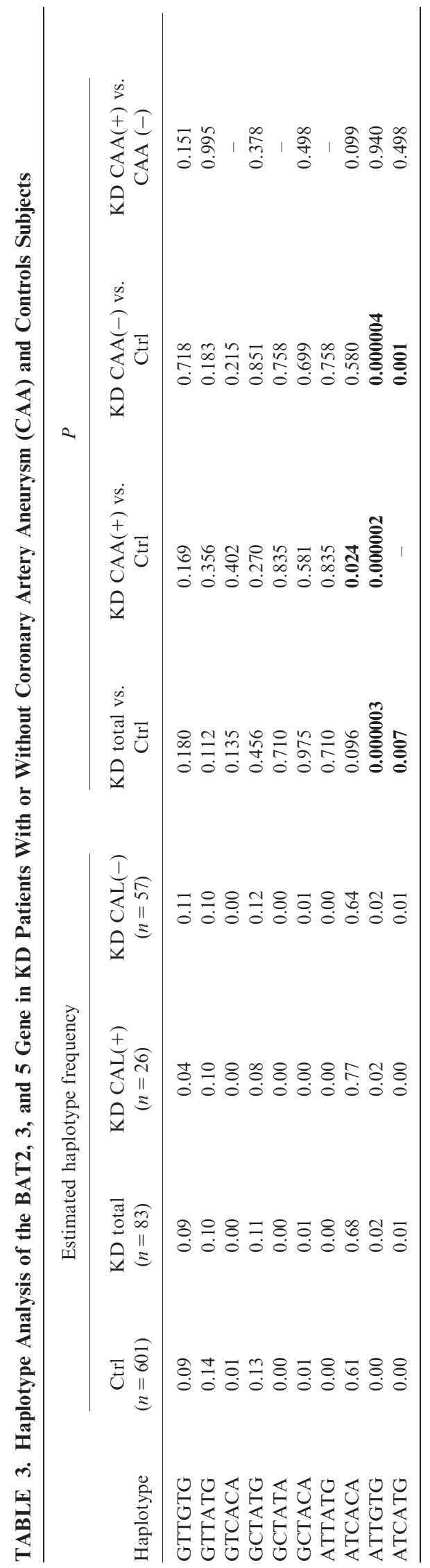

KD and CAA. The genotype and allele frequencies of BAT2, 3, and 5 polymorphisms might be useful markers for the prediction of KD or CAA susceptibility. The presences of BAT2 $-8671 * \mathrm{~A}, \mathrm{BAT} 38854 * \mathrm{C}$, and BAT5 $22655 * \mathrm{C}$ and $9569 * \mathrm{~A}$ as well as BAT haplotypes ATTGTG and ATCATG might be associated with KD or CAA susceptibility. Our findings also provide the database for further survey of BAT gene polymorphisms in other disorders. However, the real roles of the BAT polymorphisms upon the KD remain to be clarified. Furthermore, other HLA gene polymorphisms upon KD development merit further surveys.

\section{REFERENCES}

1. Kamizono S, Yamada A, Higuchi T, Kato H, Itoh K. Analysis of tumor necrosis factor-alpha production and polymorphisms of the tumor necrosis factor-alpha gene in individuals with a history of Kawasaki disease. Pediatr Int 1999;41:341-345.

2. Leung DY, Cotran RS, Kurt-Jones E, Burns JC, Newburger JW, Pober JS. Endothelial cell activation and high interleukin-1 secretion in the pathogenesis of acute Kawasaki disease. Lancet 1989;2:1298-1302.

3. Danese S, Dejana E, Fiocchi C. Immune regulation by microvascular endothelial cells: Directing innate and adaptive immunity, coagulation, and inflammation. J Immunol 2007;178:6017-6022.

4. Pay S, Simsek I, Erdem H, Dinc A. Immunopathogenesis of Behcet's disease with special emphasize on the possible role of antigen presenting cells. Rheumatol Int 2007;27:417-424.

5. Coupel S, Moreau A, Hamidou M, Horejsi V, Soulillou JP, Charreau B. Expression and release of soluble HLA-E is an immunoregulatory feature of endothelial cell activation. Blood 2007;109:2806-2814.

6. Huang Y, Lee YJ, Chen MR, et al. Polymorphism of transmembrane region of MICA gene and Kawasaki disease. Exp Clin Immunogenet 2000;17:130-137.

7. Akagi T, Rose V, Benson LN, Newman A, Freedom RM. Outcome of coronary artery aneurysms after Kawasaki disease. J Pediatr 1992;121:689-694.

8. Hung SI, Chung WH, Liou LB, et al. HLA-B $* 5801$ allele as a genetic marker for severe cutaneous adverse reactions caused by allopurinol. Proc Natl Acad Sci USA 2005;102:4134-4139.

9. Ricci E, Malacrida S, Zanchetta M, Montagna M, Giaquinto C, De Rossi A. Role of beta-defensin-1 polymorphisms in mother-tochild transmission of HIV-1. J Acquir Immune Defic Syndr 2009;51:13-19.

10. Burns JC, Glode MP. Kawasaki syndrome. Lancet 2004;364:533-544.

11. Tse SM, Silverman ED, McCrindle BW, Yeung RS. Early treatment with intravenous immunoglobulin in patients with Kawasaki disease. J Pediatr 2002;140:450-455.

12. Lin $\mathrm{YJ}$, Wan $\mathrm{L}, \mathrm{Wu} \mathrm{JY}$, et al. HLA-E gene polymorphism associated with susceptibility to Kawasaki disease and formation of coronary artery aneurysms. Arthritis Rheum 2009;60:604-610.

13. Lin CY, Lin CC, Hwang B, Chiang B. Serial changes of serum interleukin-6, interleukin-8, and tumor necrosis factor alpha among patients with Kawasaki disease. J Pediatr 1992;121:924-926.

14. Kato H, Sugimura T, Akagi T, et al. Long-term consequences of Kawasaki disease. A 10- to 21-year follow-up study of 594 patients. Circulation 1996;94:1379-1385. 


\section{Hsieh et al.}

15. Muta H, Ishii M, Egami K, et al. Early intravenous gammaglobulin treatment for Kawasaki disease: The nationwide surveys in Japan. J Pediatr 2004;144:496-499.

16. Oh JH, Han JW, Lee SJ, et al. Polymorphisms of human leukocyte antigen genes in korean children with Kawasaki disease. Pediatr Cardiol 2008;29:402-408.

17. Kim JJ, Hong SJ, Hong YM, et al. Genetic variants in the HLA-G region are associated with Kawasaki disease. Hum Immunol 2008;69:867-871.

18. Barron KS, Silverman ED, Gonzales JC, St Clair M, Anderson K, Reveille JD. Major histocompatibility complex class II alleles in Kawasaki syndrome - lack of consistent correlation with disease or cardiac involvement. J Rheumatol 1992;19:1790-1793.

19. Gnjec A, D'Costa KJ, Laws SM, et al. Association of alleles carried at TNFA -850 and BAT1 -22 with Alzheimer's disease. J Neuroinflammation 2008;5:36.

20. Ramasawmy R, Cunha-Neto E, Fae KC, et al. BAT1, a putative anti-inflammatory gene, is associated with chronic Chagas cardiomyopathy. J Infect Dis 2006;193:1394-1399.

21. Diakite M, Clark TG, Auburn S, et al. A genetic association study in the Gambia using tagging polymorphisms in the major histocompatibility complex class III region implicates a HLA-B associated transcript 2 polymorphism in severe malaria susceptibility. Hum Genet 2009;125:105-109.
22. Migita O, Noguchi E, Koga M, et al. Haplotype analysis of a $100 \mathrm{~kb}$ region spanning TNF-LTA identifies a polymorphism in the LTA promoter region that is associated with atopic asthma susceptibility in Japan. Clin Exp Allergy 2005;35:790-796.

23. Martinez A, Salido M, Bonilla G, et al. Association of the major histocompatibility complex with response to infliximab therapy in rheumatoid arthritis patients. Arthritis Rheum 2004;50:1077-1082.

24. Kwak JH, Kim SI, Kim JK, Choi ME. BAT3 interacts with transforming growth factor-beta (TGF-beta) receptors and enhances TGF-beta1-induced type I collagen expression in mesangial cells. J Biol Chem 2008;283:19816-19825.

25. Simhadri VR, Reiners KS, Hansen HP, et al. Dendritic cells release HLA-B-associated transcript-3 positive exosomes to regulate natural killer function. PLoS ONE 2008;3:e3377.

26. Pogge von Strandmann E, Simhadri VR, von Tresckow B, et al. Human leukocyte antigen-B-associated transcript 3 is released from tumor cells and engages the NKp30 receptor on natural killer cells. Immunity 2007;27:965-974.

27. Sasaki T, Gan EC, Wakeham A, Kornbluth S, Mak TW, Okada H. HLA-B-associated transcript 3 (Bat3)/Scythe is essential for p300mediated acetylation of p53. Genes Dev 2007;21:848-861.

28. Kwok PY, Gu Z. Single nucleotide polymorphism libraries: Why and how are we building them? Mol Med Today 1999;5:538-543. 